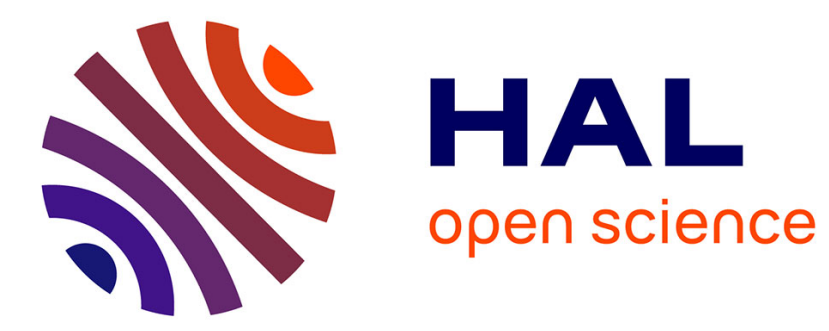

\title{
Approximate programming of magnetic memory elements for energy saving
}

N. Locatelli, A.F. Vincent, S. Galdin-Retailleau, J-O Klein, D. Querlioz, J-O. Klein

\section{- To cite this version:}

N. Locatelli, A.F. Vincent, S. Galdin-Retailleau, J-O Klein, D. Querlioz, et al.. Approximate programming of magnetic memory elements for energy saving. 2015 International Conference on Memristive Systems (MEMRISYS), Nov 2015, Paphos, Cyprus. 10.1109/MEMRISYS.2015.7378400 • hal01832350

\section{HAL Id: hal-01832350 \\ https://hal.science/hal-01832350}

Submitted on 7 Jul 2018

HAL is a multi-disciplinary open access archive for the deposit and dissemination of scientific research documents, whether they are published or not. The documents may come from teaching and research institutions in France or abroad, or from public or private research centers.
L'archive ouverte pluridisciplinaire HAL, est destinée au dépôt et à la diffusion de documents scientifiques de niveau recherche, publiés ou non, émanant des établissements d'enseignement et de recherche français ou étrangers, des laboratoires publics ou privés. 


\title{
Approximate Programming of Magnetic Memory Elements for Energy Saving
}

\author{
N. Locatelli*, A.F. Vincent, S. Galdin-Retailleau, J-O. Klein and D. Querlioz \\ Institut d'Electronique Fondamentale, UMR CNRS 8622, University of Paris-Sud, 91405 Orsay, France \\ *e-mail: nicolas.locatelli@u-psud.fr
}

The high density of on-chip nonvolatile memory provided by memristive elements is highly desirable for many applications. However, it raises concerns about finding the best programming strategies to limit the energy consumption of such systems. Here, we highlight the case of magnetic memory, where several unconventional programming strategies can reduce energy consumption, especially for applications in neuromorphic computing.

\section{STT-MTJ programming}

Magnetic Tunnel Junctions programmed through the Spin Transfer Torque effect (STT-MTJs), the basic cells of spin transfer torque magnetic RAM (STT-MRAM), feature fast programming, non-volatility and outstanding endurance. The behavior of such device is presented in Fig. 1 and is reminiscent of a binary bipolar memristor. The specificity of such devices appears through the stochastic nature of their programming. Indeed, under a programming pulse of given voltage $V_{\text {prog }}$ and duration $\Delta t$, the STT-MTJ has a non- $100 \%$ probability $P\left(V_{\text {prog }}, \Delta t\right)$ to switch state (Fig. 2). This effect has been extensively studied and the switching statistics can be described through comprehensive analytical models [1]. We consider a STT-MTJ corresponding to a $32 \mathrm{~nm}$ technology. For any voltage amplitude, models allow us to derive the reduction of the programming bit error rate (ER) - the probability of failed switching - as the pulse duration (Fig. 3) and the programming energy (Fig. 4) increase. It appears that the pulse duration gives us an efficient handle to tune the ER. Different programming regimes can then be identified depending on targeted application and error rate. Starting from a programming regime for high-significance data, ensuring $E R=10^{-10}$, we evaluate the energy reduction that can be obtained by accepting an increase of the error rate (Fig. 5).

\section{Disciplined approximate storage}

Given a programming voltage $V_{\text {prog }}=0.65 \mathrm{~V}$, allowing an increase of the ER to $10^{-2}$ grants a reduction of $62 \%$ of the energy expense. Considering the use of a STT-MRAM to store synaptic weights as floating point number in neural networks applications, strategies of disciplined approximate programming can be enforced to reduce the global energy expense [2]: identifying lower-significance part of the data i.e., least significant bits of the weight (LSB), and releasing them from low error-rate constraints. On Fig. 6, we expose the predicted energy reduction factor as a function of the number of bits labelled as lowly significant, and the error rate with which they are programmed. For instance, the reasonable choice of 26
LSB (half of the float significand) with $\mathrm{ER}=10^{-2}$ grants a $22 \%$ energy reduction when compared to a fully highsignificance memory use.

\section{MTJs as stochastic synapses}

Further increase of the ER above $10^{-2}$ corresponds to entering a regime of stochastic programming of the devices. This very low energy regime can be implemented to achieve memristive synapses with stochastic plasticity in hardware neural network $[3,4]$. In such applications, the devices state -thus the synapses conductance- evolves during the learning process according to a stochastic rule, with very high ER, possibly over $90 \%$. In that case, the drastic reduction of the energy expense ( $86 \%$ ) comes from exploiting the intrinsic device randomness as an essential feature of the system.

In recent work [3], we highlighted that a STT-MTJ based hardware neural network has potential to achieve unsupervised learning by testing it against a task of vehicle counting (Fig. 7).

\section{Sensibility to programming variability}

As can be seen on Fig. 4, the ER resilience to programming conditions variability (5\% variability on the voltage are considered here) increases as the targeted error-rate increases. While releasing low error-rate constraints, systems then tends to become more robust to variability. Notably, neural networks based on stochastic STT-MTJs with ER $=90 \%$ proved unchanged performances for devices variability up to $17 \%$ [3].

\section{Conclusion}

Deterministic programming strategies are associated to high energy cost and do not suit ideally bio-inspired applications. By contrast, strategies can be developed to consider higher ER programming for energy reduction.

\section{Acknowledgements}

This work is supported by Labex NanoSaclay (ANR-10LABX-0035), ANR COGNISPIN (ANR-13-JS03-000401), and FP7 ICT BAMBI (FP7-ICT-2013-C).

\section{References}

[1] A.F. Vincent et al., "Analytical macrospin modeling of the stochastic switching time of spin-transfer torque devices", IEEE Trans. Electron Devices, vol. 62 (1), p.164 (2014).

[2] A. Sampson et al., "Approximate storage in solid-state memories”, ACM Trans. Comput. Syst., vol. 32(3) (2014).

[3] A.F. Vincent et al., "Spin-transfer torque magnetic memory as a stochastic memristive synapse for neuromorphic systems", IEEE Trans. Biomed. Circuits Syst., vol. 9 (2), p.166 (2015).

[4] D. Querlioz et al., "Bioinspired programming of memory devices for implementing an inference engine", Proc. IEEE, vol. 103(8), pp. 1398-1416 (2015). 


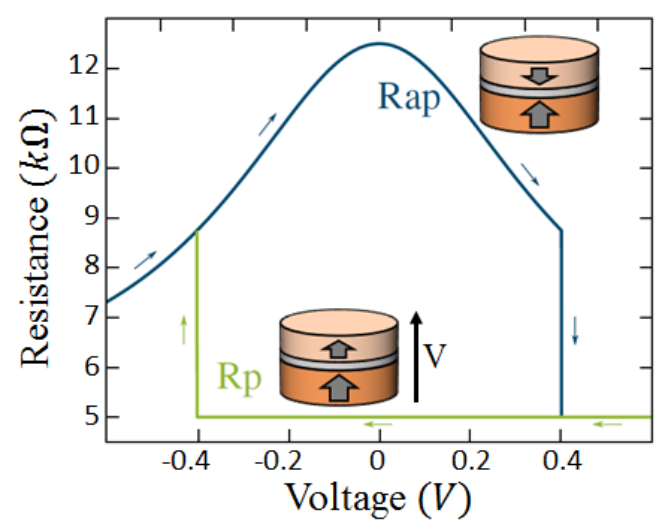

Fig. 1. DC resistance versus voltage for a magnetic tunnel junction (MTJ), composed of a stack of two magnetic layers separated by a tunnel barrier. The resistance of the device relates to the relative orientation of their magnetizations. The magnetization of the free layer is switched through the spin transfer torque (STT) effect.

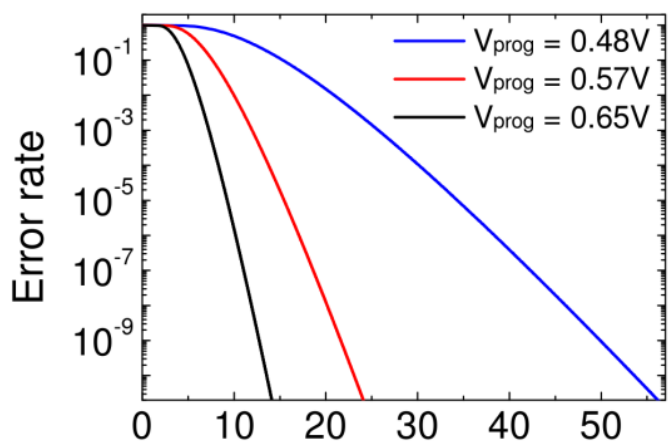

Programming pulse duration (ns)

Fig. 3. Error rate versus programming pulse duration $\Delta \boldsymbol{t}$ for different pulse amplitudes $\boldsymbol{V}_{\text {prog }}$.

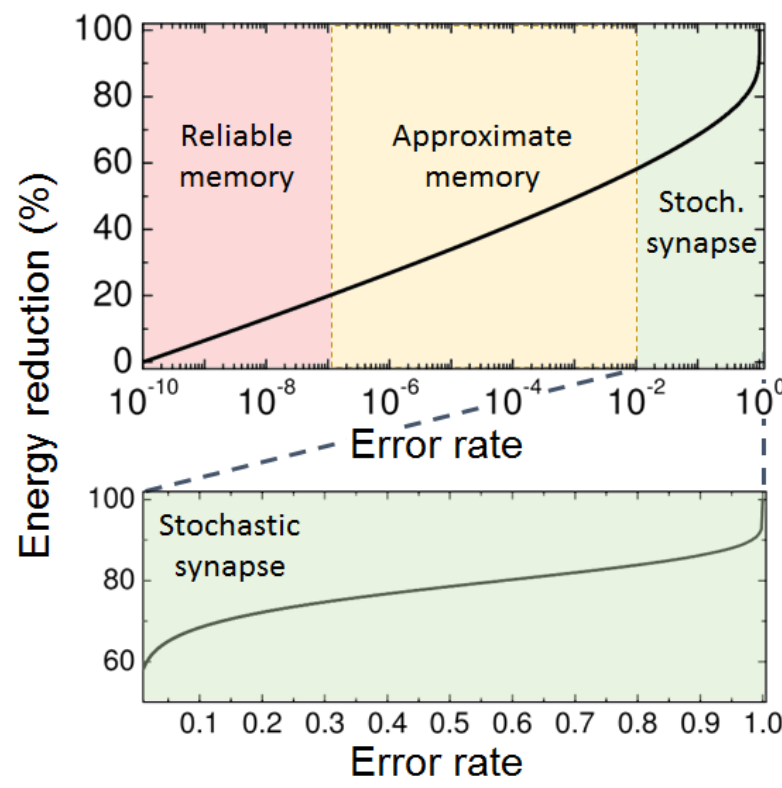

Fig. 5. Programming energy reduction factor when considering an increase of the error rate from $E R=10^{-10}$, calculated for programming voltage $\boldsymbol{V}_{\text {prog }}=0.65 \mathrm{~V}$.

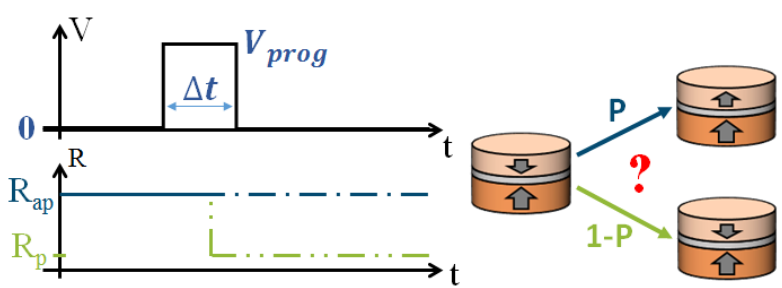

Fig. 2. Illustration of MTJ programming. After applying a pulse of amplitude $\boldsymbol{V}_{\text {prog }}$ and duration $\Delta \boldsymbol{t}$, the MTJ has a non$100 \%$ probability $\boldsymbol{P}$ to have successfully switched state.

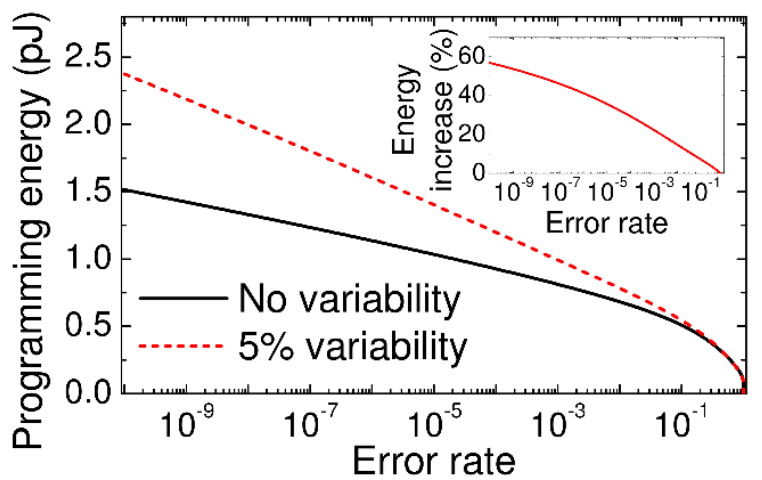

Fig. 4. Error rate versus average programming energy calculated for programming voltage $\boldsymbol{V}_{\text {prog }}=0.65 \mathrm{~V}$ and considering $5 \%$ variability on programming voltage. Inset shows the energy increase due to variability.

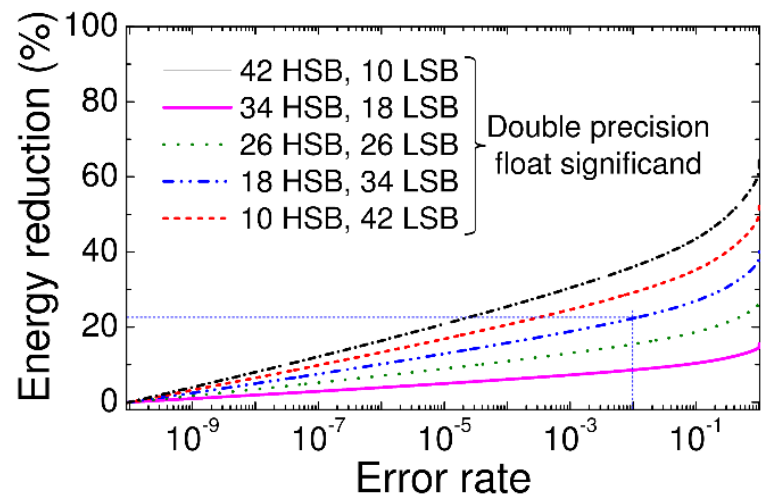

Fig. 6. Energy reduction of the total programming energy for a double precision float considering an increase of the ER for low-significance bits (LSB). Different choices for the number of LSB and high-significance bits (HSB) are presented.

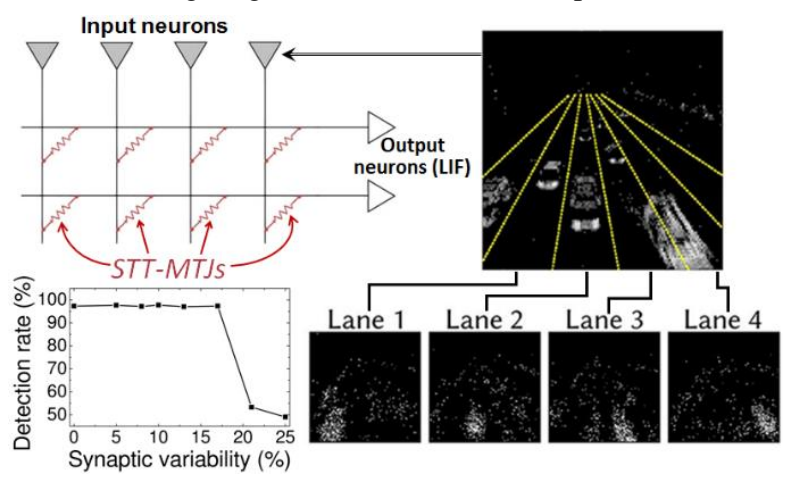

Fig. 7. Hardware neural network architecture with STT-MTJs as stochastic synapses. The system is evaluated against a car detection task, proving $95 \%$ detection rate and high resilience to synaptic variability (bottom left inset). 